\title{
Immunologische Assays im Vergleich zum Hauttest
}

\author{
Interferon-y-Release-Assays (IGRA) stellen eine sensitive \\ Alternative zum Tuberkulin-Hauttest (TST) dar. Ob sich der \\ Einsatz zur Routinekontrolle auf eine latente Tuberkulose- \\ Infektion in Gesundheitsberufen bei insgesamt niedriger \\ Inzidenz eignet, haben Susan E. Dorman et al. untersucht. \\ Am J Respir Crit Care Med 2014; 189: 77-87
}

Die Querschnittstudie mit longitudinaler Weiterbeobachtung umfasste 2563 bisher nicht an Tuberkulose (TB) erkrankte Personen aus Gesundheitsberufen, die in einem von 4 US-amerikanischen Gesundheitszentren beschäftigt waren. Die Tuberkuloserate in den betreffenden Regionen lag bei 4-9/100000 Personen. Für das Screening wurden die IGRAs QuantiFERON-TB-Gold-In-Tube (QFTGIT) und T-SPOT.TB (T-SPOT) sowie der TST zu Beginn und alle 6 Monate für insgesamt 18 Monate eingesetzt. 9\% der Studienpopulation gaben an, gegen Bacillus Calmette-Guérin (BCG) geimpft zu sein, was vorrangig im Hauttest, nicht aber in den Immuntests zu falsch positiven Befunden führen kann.

Am Basistest nahmen 2418 Personen teil. $\mathrm{Zu}$ diesem Zeitpunkt ergab sich im TST bei 5,2\% der Teilnehmer ein positives Ergebnis ( $n=125)$, im QFT-GIT bei $4,9 \%$ $(\mathrm{n}=118)$ und im T-SPOT bei $6,0 \%(\mathrm{n}=144)$. War der TST positiv, eine IGRA aber negativ, war dies klar assoziiert mit einer BCG-Impfung (Odds Ratio = 25,1 vs. keine BCG-Impfung; 95\%-Konfidenzintervall 15,5-40,5).

\section{Deutlich mehr positive Befunde $\nabla$}

Im Rahmen der 18 Monate dauernden Beobachtungszeit ergab sich kumulativ bei 138 von 2263 Teilnehmern (6,1\%) eine Konversion des Ergebnisses im QFTGIT, bei 177 von 2137 (8,3\%) bei Anwendung des T-SPOT und bei 21 von 2293 $(0,9 \%)$ im TST. Damit unterschieden sich die Konversionsraten zwischen den Tests statistisch signifikant $(p<0,001$ für den Vergleich von QFT-GIT mit TST und für T-
SPOT vs. TST; $\mathrm{p}=0,005$ beim Vergleich QFT-GIT vs. T-SPOT). Bei keinem Teilnehmer zeigte sich in allen 3 Tests zum gleichen Zeitpunkt eine Konversion. Es lag auch keine signifikante Assoziation der Konversion mit einer TB-Exposition vor.

Im Falle einer Konversion in einem der immunologischen Tests waren die Ergebnisse 6 Monate später in mehr als 3 Viertel der Fälle wieder negativ: beim QFTGIT bei 81 von 106 Betroffenen (76,4\%) und bei T-SPOT bei 91 von 118 zuvor positiv Getesteten (77,1\%). Wurden die Tests 2 Wochen nach erstmaliger Konversion noch einmal durchgeführt, ergab sich bei 15 von 170 Teilnehmern $(8,8 \%)$ im QFT-GIT eine Diskordanz, bei T-SPOT war dies bei 19 von 151 (12,6\%) der Fall.

\section{Fazit}

Die hier erhobenen Daten zeigen eine geringere Spezifität der IGRA in Niedrig-Risiko-Gebieten als bisher angenommen: Bei geringer Tuberkuloseinzidenz sind die meisten mit IGRA detektierten Konversionen falsch-positiv. Solche falsch positiven Ergebnisse waren mit den IGRA 6-9 Mal häufiger als mit dem TST. Die erneute Testung von Personen mit Konversionen ist daher dringend anzuraten. Insgesamt stellen diese Ergebnisse die Routinetestung von Personen in Gesundheitsberufen in Niedriginzidenzgebieten überhaupt in Frage, so die Autoren.

Friederike Klein, München
Forschung

\section{Gewappnet für den ersten Atemzug}

Frühgeborene haben ein erhöhtes Risiko, an Funktionsstörungen der Lunge zu erkranken. Die Ursache dafür liegt u.a. in der unvollständigen Lungenreifung während der embryonalen Entwicklung. Bei einer drohenden Frühgeburt versucht man daher, die Lungenreifung zu forcieren. Therapeutische Ansätze für eine induzierte Lungenreifung vor der Geburt beschränken sich hauptsächlich auf die Gabe von Glukokortikoiden, z.B. Betamethason. Wie die Prozesse der embryonalen Lungenreifung auf der molekularen Ebene genau vonstatten gehen, ist bislang nur unvollständig verstanden.

Die Wissenschaftler um Prof. Jens Kroll, Mannheim, haben jetzt im Rahmen einer experimentellen Studie festgestellt, dass Blutgefäße eine wichtige, aktive Funktion bei der embryonalen Lungenreifung haben. Bei ihren Untersuchungen am Mausmodell konnten sie zudem einen molekularen Schalter identifizieren, der durch Betamethason aktiviert wird. Die Ergebnisse erschienen im Mai in der Fachzeitschrift Disease Models \& Mechanisms. Die Autoren konnten 2 Proteine identifizieren, die eine wichtige Rolle bei der Lungenreifung spielen. Sie werden als KLEIP und HIF- $2 \alpha$ bezeichnet. Fehlen diese oder sind zu wenig davon in der Lunge vorhanden, so können embryonale Lungenreifungsprobleme auftreten, die nach der Geburt zu Atemproblemen führen. Es konnte gezeigt werden, dass Betamethason gezielt die Menge an HIF- $2 \alpha$ in den Lungen erhöhen kann, was zu einer verbesserten embryonalen Lungenreifung und Lungenfunktion nach der Geburt führt.

Die Mannheimer Wissenschaftler haben mit KLEIP und HIF-2 $\alpha$ also einen wichtigen Regulator der vorgeburtlichen Lungenreifung identifiziert, welcher durch Betamethason aktiviert wird. Wenn es gelingt, über die gezielte Aktivierung dieses Schalters die Lungenreifung effektiv steuern zu können, kommt man dem Ziel, eine häufige Erkrankung von Frühgeborenen, das sog. Atemnotsyndrom zu verhindern, einen wichtigen Schritt näher.

Nach einer Mitteilung der

Universitätsmedizin Mannheim 